\title{
Shaping the Next Generation Pharmaceutical Supply Chain Control Tower with Autonomous Intelligence
}

\author{
Matthew Liotine
}

Information \& Decision Sciences, University of Illinois at Chicago

\begin{abstract}
This paper summarizes the findings of an industry panel study evaluating how new Autonomous Intelligence technologies, such as artificial intelligence and machine learning, impact the system and operational architecture of supply chain control tower (CT) implementations that serve the pharmaceutical industry. Such technologies can shift CTs to a model in which real-time information gathering, analysis, and decision making are possible. This can be achieved by leveraging these technologies to better manage decision complexity and execute decisions at levels that cannot otherwise be managed easily by humans. Some of the key points identified are in the areas of the fundamental capabilities that need to be supported and the improved level of decision visibility that they provide. We also consider some the challenges in achieving this, which include data quality and integrity, collaboration and data sharing across supply chain tiers, cross-system interoperability, decision-validation and organizational impacts, among others.

Keywords: Supply Chain Management; Control Tower; Autonomous Intelligence
\end{abstract}

ARTICLE INFO

Received: Jan 29, 2019

Accepted: Mar 11, 2019

Available online: May 15, 2019

*CORRESPONDING AUTHOR

Matthew Liotine, Information \&

Decision Sciences, University of

Illinois at Chicago;

mliotine@uic.edu;

\section{CITATION}

Matthew Liotine. Shaping the Next Generation Pharmaceutical Supply Chain Control Tower with

Autonomous Intelligence. Journal of Autonomous Intelligence 2019; 2(1):

56-71. doi: 10.32629/jai.v2i1.34

\section{COPYRIGHT}

Copyright (C) 2019 by author(s) and Frontier Scientific Publishing. This work is licensed under the Creative Commons

Attribution-NonCommercial 4.0 International License (CC BY-NC 4.0).

https://creativecommons.org/ licenses/by-nc/4.0/

\section{Introduction}

Today's pharmaceutical distributors are faced with several key strategic priorities. These include retaining and managing operating margin, acquiring business agility and controlling pharmaceutical counterfeiting and fraud. Overall, the control tower (CT) concept can transform how healthcare and pharma industries lead and manage their supply chains by shifting to a model in which real-time information gathering, analysis, and decision making are possible. In essence, a CT is a center of excellence that facilitates a coordinated network to continuously manage complexity and execute at levels that cannot otherwise be managed easily by humans. It must provide fundamental capabilities to enable the levels of visibility and awareness to achieving this mission (1).

This research was a result of an industry study whereby a panel of experts was assembled to derive an accelerated view on the future of control tower supply chain capabilities within the pharmaceutical industry. The emphasis of the study was to determine what form the $\mathrm{CT}$ of the future would take, and to identify those areas within the CT framework that would be most fertile for application of forwardlooking Autonomous Intelligence (AI) related technologies. A range of needs was explored by obtaining unique perspectives from the panel, representing the entire pharmaceutical value chain, including those from academic, manufacturing, distribution, pharmacy and technology fields, as well as other key players in the ecosystem (2).

Today's CT solutions provide somewhat simplistic, limited operational views while claiming end to end visibility, providing a window into certain parts of the 
supply chain at the expense of others (3). Ultimately, visibility boils down to the execution of orders while balancing costs and availability to satisfy contracts and customer demand. In this respect, the CT should provide a holistic end-to-end view across supply chain suppliers, contract manufacturers, transportation carriers, and third-party logistic vendors to track the active movements of goods from origin to destination (4) . To this end, the panel explored the common levels of visibility that are required by pharmaceutical distributors and the degree of visibility needed both now and within the future, with the understanding that visibility can vary relative to the supply chain tier, type of supplier and type product or product group.

Overall, a CT serves as a command center to enable a firm to act more closely with suppliers and be more proactively provide customer service, and ultimately improve profitability. Figure 1 conveys the traditional view of the functional architecture of a CT (5).

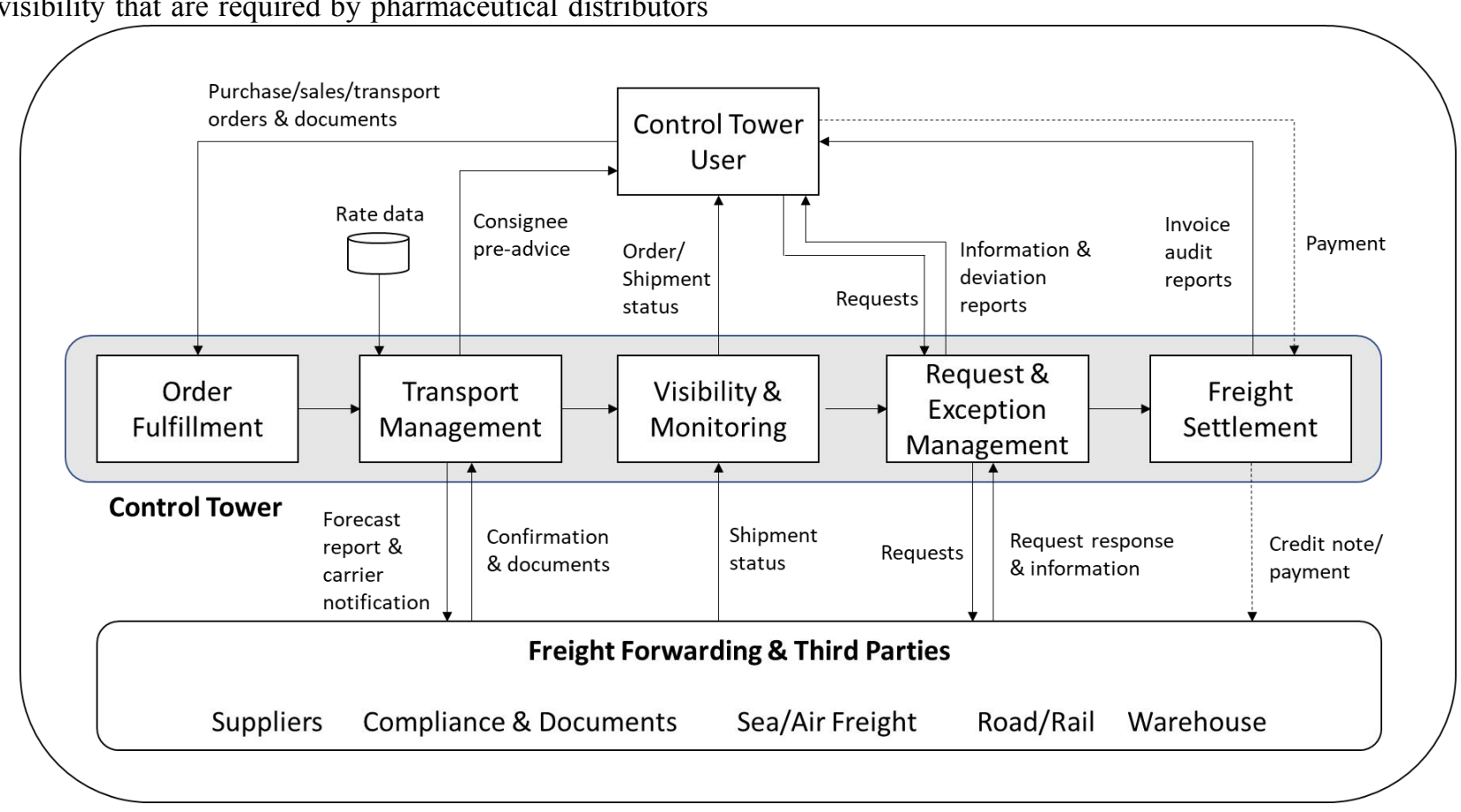

Figure 1. Conventional View of Supply Control Tower Functional Architecture

\section{Fundamental CT Capabilities}

The CT must provide capabilities that are shared across supply chain players and third-party providers. These include:

- Data - A CT must enable the free flow access and sharing of data among supply chain players, including third-party providers. External data, financial and transactional information must be managed effectively to make real-time recommendations and decisions. The information should be timely, enabling real-time decision making in response to potential disruptions (6).

- Alerting - The CT should have the ability to identify bottlenecks, deviations and exceptional events within daily operations and provide trigger information such that resources can be redirected to maintain desired service levels. The CT should facilitate alerts on an individual event or periodic basis, such that the controller not only can manage the situation and arrive at a positive outcome, but can also provide the supply chain and logistics planning functions with actual performance data to drive decision making (7).

- Operational functions - The CT should facilitate the core supply chain operational functions of plan, source, make and deliver, relative to their physical and logical execution. The CT should enable monitoring supply chain operations and identify variances between actual performance and service levels agreed with customers, as well as monitoring internal key performance indicators (KPIs), such as working capital investment, lead time, and quality 
indicators, among others (8).

- Automation - Decision processes should be automated through the CT, to a point where they can be placed on autopilot and be auto-corrective, minimizing the need for manual processes for response and event intervention.

- Decision support - Decisions within a CT environment should be made more proactive in nature, through monitoring dashboards and information received from internal and external sources such as suppliers and customers. There is an ongoing need for using predictive and prescriptive analytics to enhance current decision-making processes.

- $\quad$ Role transformation - A CT enabled with AI should move practitioners from serving as supply chain, logistics, and customer service managers towards the role of specialists in order, supply and logistics fulfillment.

Accomplishing the above is not without some key challenges. The following are some of the challenges that were identified:

- There could be many tiers of the supply chain involved in a CT, so at the outset tiers should be identified based on the criticality of the participants that are the most important.

- Data quality and information can be flawed or inadequate to measure certain aspects of the distribution channel. This will require some internal cross functional alignment and agreement between players to capture and manage the necessary data. Data volumes can be massive if managing at the stocking unit (SKU) level, requiring methods for preprocessing and consolidating information.

- Integrating the planning and execution between organizations requires significant work to sort through information across multiple systems with varying technologies. Scalability becomes more difficult when trying to customize interfaces with trading partners, which may not be reusable.

- Foundational work within the organization will be required to build the basis for standardizing manual processes, such that they can be automated with enabling software and technology. This could eliminate some of the human intervention that is often required in transactional processes.

- Technology innovation in the life science industries can be challenging due to the conservative and reactive nature of the industry. Process changes can be costly due to process revalidation and the discrepancies of embracing such changes across partners.

Altogether, a CT should enable a win-win situation across the distribution network, allowing the distributor and other players to perform at optimal levels. To this end, there are many pre-requisites to implementing a CT solution. Among them are the development of a commercial plan to highlight new business models or changes in aspired service levels and volumes, on both an enterprise and customer or category level, based on criteria such as volume and/or strategic importance. In addition, a deep understanding of customer product handling is needed to leverage supply chain risk sharing business models at customer sites such as consignment stock management, inventory planning, electronic data interchange (EDI) for replenishment and spare parts handling (9) . This is becoming more common in the healthcare and medical technology fields.

\subsection{Industry Trends Influencing Visibility}

Several key industry trends were identified that are driving the overarching visibility requirements of the CT:

- End-to-end patient care:

This trend is motivating partnerships among logistics firms, pharmaceutical producers, and local pharmacists to form powerful interconnected patient centric networks that are demand driven so that patients can obtain what they need and when they need it (10).

- Direct to consumer:

Large pharmaceutical distributors are moving towards a direct to consumer model, competing with entities such as Walgreens.

\section{- Competitive threats from expert supply chains:}

Strong partnerships are emerging in order to improve visibility and cooperation across the network, involving players such as Amazon.

While the primary goal is to serve the patient, they must create higher value services and with lower costs, year after year, to offset constantly low margins. The pharmaceutical distributor will have constantly changing needs for CT visibility. At the core of visibility is the 
information linked to physical product movements. Delivery pressures require them to maintain many smaller and regional centers that are contractually required to hold inventory and comply with service level agreements. The visibility and number of required tiers is commensurate with the cost and criticality of the products managed. For example, medical supplies, over the counter (OTC) products, and pharmaceuticals will require different types of visibility and network design. Furthermore, different players will need different visibility levels, and it will be incumbent upon the CT to aggregate information according to the needs of each level.

Supply chain CTs are supported by three sets of technologies in particular (11):

- Supply chain partner technologies which include enterprise resource planning (ERP) and optimization, transportation and warehouse management;

- Cloud-based technologies that connect all the nodes along the end-to-end supply chain, reducing the cost, complexity and time-to-value of inter-enterprise integration;

- Analytic technologies that enable organizations to make use of the enormous amount of data being collected across the supply chain.

\subsection{Information Flows and Channels}

Information is the media that enables visibility for the CT. There are three classes of information that were identified as necessary to underpin visibility:

- Transactional Data:

This includes standard order and transactional order data and information obtained from an ERP or transactional system that can be shared upstream and downstream.

- Regulatory Information:

CTs should have secure shared regulatory status information so as to reduce significant bullwhip effects arising from regulatory approvals occurring at different stages. Such information can trigger new demand throughout the value chain resulting in high priority surges for small quantities of development trials or large quantities for startup, launch, and order fulfillment. Both suppliers and distributors require visibility to forecast and communicate regulatory status related to their customer's regulators.

\section{- Transport Information:}

Instead of each supplier sharing their own transportation information, transport providers should also be connected with each of the other's CTs, eliminating duplication of information and providing easy access to current costs, lane capacities/capabilities, transport issues, weather events, strikes and so on. More precise lead times can be drawn from both the supply and transport base to provide more accurate real time information.

Pharmaceutical distributors will be managing and communicating supply chain information moving upstream to and from the manufacturer base, and downstream to and from the customer base (e.g. the provider or retailer), and ultimately to the customer (or patient) base. Hundreds of manufacturers and provider or retailer contracts are being managed, as well those of third-party re-packers or distributor owned re-packers. Tracking and tracing transcend both upstream and downstream flows. Although tracking and tracing require different capabilities, both are necessary for physical flow visibility. These functions can be centralized more efficiently by a CT for pharmaceutical distributors than a decentralized model, by providing distributors with the knowledge of where products came from (tracing) and where the products are going (tracking). Such directives as the US Drug Supply Chain Security Act (DSCSA) require product serialization to support these capabilities.

\subsubsection{Upstream Flows}

Upstream flows entail a tremendous amount of information from the manufacturer to the distributor. Such information can impact inventory planning at the main procurement centers and down through the enterprise to numerous regional distribution centers. Because of the nature of the bullwhip effect, it is easier to manage information and visibility going upstream, although the impact in inventory and cost is larger. Upstream levels of visibility and the number of tiers would depend on the size of the supply chain. While 1 or 2 tiers are desirable, additional tiers would help in reducing the bullwhip effect with regard to inventory levels and expected supply disruptions. Upstream levels 
of visibility and information that are typically available and desirable include:

- Inventory or product quantity in each stage, hub, vendor or player (currently available);

- Inventory in transit and expected lead times (currently available);

- Quality excursions (for instance temperature excursions, and how much of the stability budget is consumed), even if they do not impact product loss (currently available);

- On-time performance of the different players, such as on-time performance when using an associated vendor (currently available);

- Real lead times from site to site, which are often different from those promoted by suppliers and customs (desired);

- Supply interruptions and possible delays due to weather, holidays, recalls, etc. which in turn prompt information flow regarding get well dates and substitution recommendations. This is needed to plan reposts and to provide alternatives as part of the service (desired);

- Demand for specific customizations or configurations (desired);

- Demand patterns from the customers (i.e. locations that have more or less elasticity to price and supply changes, or those that tend to aggregate purchases on particular days, etc.) (desired);

- Changes in costs which can prompt pricing changes (desired);

- $\quad$ Product allocations and supply distributions from the manufacturer, and downstream inventory impacts (desired);

- Product promotions and new product launch timelines and configurations/strengths available, with first to shelf generic availability information (desired) (12);

- Product discontinuations to include timelines and substitution recommendations (desired);

- $\quad$ Product recalls or required market notifications and required or recommended plans of action (desired);

- Compliance to regulation timelines such as serialization effort and any impacts on supply availability (desired) (13);

- Contract position information to include pricing position gained by the manufacturer as negotiated with the contracting organization (desired);

- New packaging and labeling product information (desired);

- Tracing information to combat counterfeit drugs, which captures where a product unit was produced, packed, stored, and shipped through all of the nodes touching the final product (referred to as provenance). This requires the CT to maintain a centralized data model that could be could be unit level relational or nested. Visibility tracing begins from the point of the last data capture and ends upstream at the point of production (desired).

\subsubsection{Downstream Flows}

Downstream information flows should be tailored based on the specific type of customer or contract (e.g. end-retailer, pharmacy, hospital, etc.). Downstream players will require similar information provided to the distributor from the upstream path, but in a different format depending on the contract being administered to that specific provider or retailer. Downstream levels of visibility and information that are typically available and desirable would include:

- $\quad$ Product recalls alerted to the end pharmacy;

- Quality excursions alerted to the end pharmacy;

- Inventory in transit, locations and lead times (desired);

- $\quad$ Real lead times, such as those based on the average of the last several trips on a particular route(desired);

- Supply interruptions, which would be of particular interest to large retailers so that they could redirect inventory from other locations (desired);

- Batch expiration and information issued to the end pharmacy;

- $\quad$ Price elasticity, especially noted increases (desired);

- Demand patterns, especially noted increases (desired);

- Penalty payments, prompted by supply chain performance and reconciliation to penalty claims (desired);

- $\quad$ Third party fee forecasts (desired);

- Tracking information that identifies the current and future product locations and destination arrival times. This can help ensure promptness of 
delivery, but may require technology to merge real time data with milestone data interacting with the transportation (TMS) and warehouse management system (WMS) of the various players in the supply chain (desired).

In addition, customers or patients represent the point of consumption of downstream information, and will require a visibility level similar to that of the consumer-packaged goods industry. Among such information would be the availability of medications and supplies, critical issues such as recalls or contamination, and medication alternatives that are better or cost effective.

\subsection{CT Visibility Challenges}

All of the above is not without challenge. Those challenges that were noted as being the most significant in limiting CT visibility include:

- Limitations in technology, such as serialization, can impact CT visibility and how smoothly information flows in a heavily data-integrated environment. Blockchain technology has the potential of facilitating access and updates regarding product movements to supply chain players without a central intermediary. However, as in the centralized intermediary model, it requires significant interconnectivity between systems.

- The lack of some level of regulation or governance, which is desirable to regulate interaction and avoid conflicts of interest. This can also avoid information distortions in demand patterns, such as those arising from anticipated shortages, while granting visibility to all customers, irrespective of their volume of business. Regulatory constraints and lack of collaboration across the supply chain have slowed down the adoption process in the pharmaceutical industry.

- More accurate demand forecasts methods could reduce the amount of required working capital in the form of inventory needed to expedite service orders. The need is to accurately depict demand in near-real time, moving from demand forecasting to demand sensing, as seen in the fast-moving consumer goods industry.

\section{Decision Capabilities}

The supply chain CT must support decision-making and controls for operators in a pharmaceutical distribution environment. In addition to visibility and alerting capabilities, CTs must provide operators with the ability to make decisions and execute measures in response to operational performance issues and disruptions. In this respect, the following were some of the issues addressed in the study:

- The most common types of decisions that operators need to make, and at what levels (e.g. field, transactional, executive, others);

- Similarly, the most common types of controls that operators need to execute, both now and in the future, and at what levels;

- The kinds of mechanisms the CT must provide to support these actions;

- The most effective or desirable ways of communicating remedial actions to partners through a CT, particularly edge partners, such as pharmaceutical suppliers or logistic service providers, through a CT;

- The challenges in establishing these mechanisms and how could they be overcome.

The essential capabilities of a CT are visibility, analytics and execution. CTs should provide information to support decisions at all levels, with different modes and frequencies of relationships between $\mathrm{CT}$ operators and clients. CT operators must align with clients prior to making decisions, and understand the controls that a client might require to make even the most transactional choices in a fast and effective way. The following describes some cited capabilities at different levels.

\subsection{Operational/Execution Level Capabilities}

Controls at this level could include smaller impact decisions, without major financial impact, with affirmation from CT operators. Decisions should be enacted using immediate response controls with a pool of options. Definitions on the scope of provider responsibility can help to automate communication of remedial actions. The following capabilities were identified:

- Event base alerts at the transaction level - issuing 
alerts to specific players when an event occurs, or if an event did not occur within a planned timeframe. The alerts should influence decisions such as:

- Who will ship product loads?

- What batches should be shipped?

- When can an order be picked and shipped?

- Do additional resources need to be booked for receiving a truck?

- Notification of any product recalls or quality concerns;

- Delays and/or changes in orders.

- $\quad$ Track and trace to provide supply chain physical visibility - automated to the extent possible, due to the massive volumes of transactions. Quality excursions should indicate who needs to take action, how often they be reviewed and should be incorporated into the performance metrics. Quality issues downstream can cause stoppages.

- Controls over budget allocation - for unplanned incidences or orders;

- Rush order handling process - for unplanned customer orders within a production cycle. This should include:

- Ability to analyze different response execution options, including placing it on back order;

- Impacts on other committed orders;

- Rescheduling committed orders based on the response and the effect on back-orders;

- Cost and profit impacts on supply chain KPIs for each response option.

This level would require extensive automation using rule engines, transaction processing, and work flow management. Another enabling technology is required to allow CTs to interact directly without the need for email. Technology such as smart contracts can instantly enable the communication of remedial actions. Cloud based technologies and "as-a-service" models can offset initial capital investments, since they can simplify implementation and unite more partners on a common platform, enabling real time data availability and accuracy from end-to-end.

\subsection{Tactical Level Capabilities}

The CT must provide mid-level managers with decision level capabilities to devise short-term optimal courses of actions based on analysis of the supply chain's current operational picture. These would include the following capabilities:

- An analytical/optimization engine process - to provide insights from data, particularly when demand or supply vary significantly within a planning horizon, in order to analyze the possible "what-if" options using cost versus profit and KPIs to arrive at optimized responses. Some of the decision/response areas should include:

- Reporting performance of the system:

- Status of supply for current orders;

- Order confirmations;

- Transit times;

- Current transport/logistical lead times;

- Current availability of material (un-forecasted and forecasted);

- Order policies and stock policies.

- Identifying outliers:

- Transportation concerns such as port delays, lane closures/delays, customs status of orders - export or air transport;

- Suppliers not delivering on time or/and in full.

- Noticing trends or flagging risks in the operation:

- What route should be used?

- Ship by air, road or sea?

- How is the season planned?

- What carriers are complying with their contracts?

- Visibility to inventory and upcoming orders - can support decisions regarding:

- Order expediting through other transportation means;

- Placement of additional orders to increase buffers where required;

- Communication to suppliers of changing demand patterns caused by orders or other issues;

- Allow suppliers time to react and work upstream to meet demand without the delays that cause bullwhip effects

- Available manufacturing capacity - that can be released within the planning horizon due to material unavailability and/or quality issues, or unplanned shutdowns; 
- $\quad$ Short to medium term forecast and capacity sharing - usually no more than an 18-month horizon. Enabling the ability to evaluate available options when forecasts are updated due to demand spikes or rush orders within the planning horizon.

At this level, technologies such as machine learning and/or AI could be useful.

\subsection{CT Operative Model for AI}

Effective CT implementation can present unique opportunities for driving supply chain strategies for business outcomes. These capabilities must afford executives with the following abilities:

- Longer term decision-making - concerned with the downstream ability to supply product using upstream demand visibility;

- Enable decisions to invest - where capacity is needed or new technologies are required;

- Enable the ability to move goods - to customers or from suppliers depending upon the perspective;

- Metrics on supply chain cost - such as the cost of spare capacity, logistics costs, working capital value, actual costs versus budgets adapted to market conditions, expediting costs and transparency on volume assignment costs will aid in the ability to demonstrate or reliably forecast return on investment (ROI);

- $\quad$ Performance metrics - at the strategic level such as on-time delivery performance; demand trends; capacity levels;

- $\quad$ A CT data repository for strategic decisions - a CT data base stores records of historic information needed to query and perform deep dive analysis by any player for strategic decision-making;

- A supply chain design capability - centralizing the supply chain design within the CT Operative Model. This would support the following decisions:

- How should product flow be optimized to reduce costs or achieve certain service levels?

-Where should raw materials be sourced from?

- How many DCs should are needed, including recommending new greenfield distribution centers?

- What capacity do facilities need?

- Long term forecasts - where applicable for longer term planning required to meet market trends;
- Visibility to request for quotes (RFQs) - so that organizations can appropriately respond;

- Metrics to ensure fair load designation - to prevent bad performers from receiving major or critical assignments, to ensure contracts are assigned to the best offer to the client perhaps through using authorization matrices;

- Visibility to promotional activities - to avoid bull whip effects;

- Communication to changes in regulations understanding of the trends and concerns in the transport industry. Given that the industry is highly regulated, regulatory information, particularly those that can lead to a distribution or production stoppage, is needed to allow executives to react.

Dashboard technology would help executives track the health of the value chain and react if needed to prevent supply disruptions. KPIs to include in the dashboards could be the evaluation of past CT responses to decisions and assess the dollar value. This level would necessitate human resources utilizing data analytics with different tools and models, as well as management expertise that can incorporate qualitative aspects not easily captured by models, such as corporate values, culture, mission and vision, social impact, and more.

\subsubsection{Required Organizational Capabilities}

Commitment by the entire organization, including suppliers, is key for effective decision-making and remedial action. The organization and its suppliers should be held accountable for execution of remediation activities. Senior executives should designate specific teams or personnel with sufficient technical expertise and management support to investigate quality issues and identify root causes, as well as establish lean processes.

Stakeholder contracts should require the method, timing and detailed information required for communicating remedial actions and related activities, which can vary by contract, particularly any information with the upstream partners that, if not communicated, can cause disruptions. The CT should enhance trust and collaboration among partners, such as that between customers and suppliers when faced with disruptive situations. 


\section{Technology Capabilities}

The types of technologies that are best suited to support decision-making and response activities in the CT environment were explored. The technologies that are most applicable or promising for specific kinds of decisions or control actions at the operational, tactical or strategic level were conveyed, using specific examples of actual use cases of the technology and success stories and non-success stories. The technology and operational prerequisites were envisioned for enactment within a specific decision-making context, with emphasis on systems or other technologies that are needed to be in-place or available in order to support CT operations.

\subsection{Technology Levels}

There are three levels of basic technology pre-requisites for $\mathrm{CT}$ operation that were defined:

- Data Capture \& Translation - This technology transforms physical data into digital data. Use of barcode and scanner technology are quite common, but more advanced technologies such as radiofrequency identification (RFID), smartphones, and Internet of Things (IoT) can further improve process efficiency (14).

- Data Transmission \& Upload - Moving captured data from local sources to global, cloud system or databases for CT access. Electronic data interchange (EDI) has been in use for quite some time, and application programming interface (APIs) are also popular for connecting systems. There is also a need to fully integrate Global Positioning System (GPS) technology, which can provide accurate asset location data in real time.

- Data Access \& Actionability - The CT must access the data and have the ability to perform analyses and issue alerts. Integration with other systems such as an ERP, WMS, TMS and supply chain event management systems which manage exceptional events.

The presence of a well-established ERP, truly integrated across all areas of the supply chain would be helpful. While any company could potentially outsource parts of their supply chain to operators running a CT, the presence of an established ERP system would enable the CT to leverage data for continuous process improvement.

\subsection{Levels of Application for AI}

Placement of AI technologies within the CT environment are illustrated in Figure 2 (15) . They are best described according to the levels of decision making identified in the previous discussion:

- Operational Level: This level involves near real-time decision-making and thus would involve traditional business-to-business (b2b) messaging, cloud integration and newer IoT and blockchain related technologies. These can be used collaboratively evaluating real-time executional issues and exceptions. AI can help front-line expeditors become strategic thinkers, negotiators, and decision makers rather than order expeditors (16) . AI algorithm bots can efficiently and cost-effectively automate trivial transactional tasks across these different technologies without the need for hardcoding rules, as seen with traditional software automation solutions (17). As long as processes can be characterized as procedures, AI software bots can learn to transact orders, enter forecasts and deliver information to logistics providers, among other tasks. Automating such tasks can reduce transactional costs.

- Tactical Level: This level involves evaluating multiple conditions and defining the optimum tactics and responses. AI can play an important role through its ability to employ continuous learning and adaption in making and executing decisions over shorter horizons. It can be used to automate tactical decisions by recognizing patterns, such as modifying product distribution in response to a perceived trend or bullwhip effect, scheduling freight, developing campaign schedules, issuing DC replenishment schedules, and so on.

- Strategic Level: This level will ultimately evolve to executing actions based on continuous optimization. AI technology can be embedded within decision planning systems that enable self optimization through continuous learning, developing the ability to deal with unknown situations. Bots would be able to imitate processes, evaluate options and make decisions on their own. It could potentially provide greater visibility to life cycle decisions by mining leading indicator data to identify longer 
term strategic trends or shifts in demand or supply and suggest optimal responses. The ability to react faster allows meeting windows of opportunity in recommending or acting upon sourcing, supplying, or distribution signals. Thus, AI technology might deliver its greatest value at the strategic level.

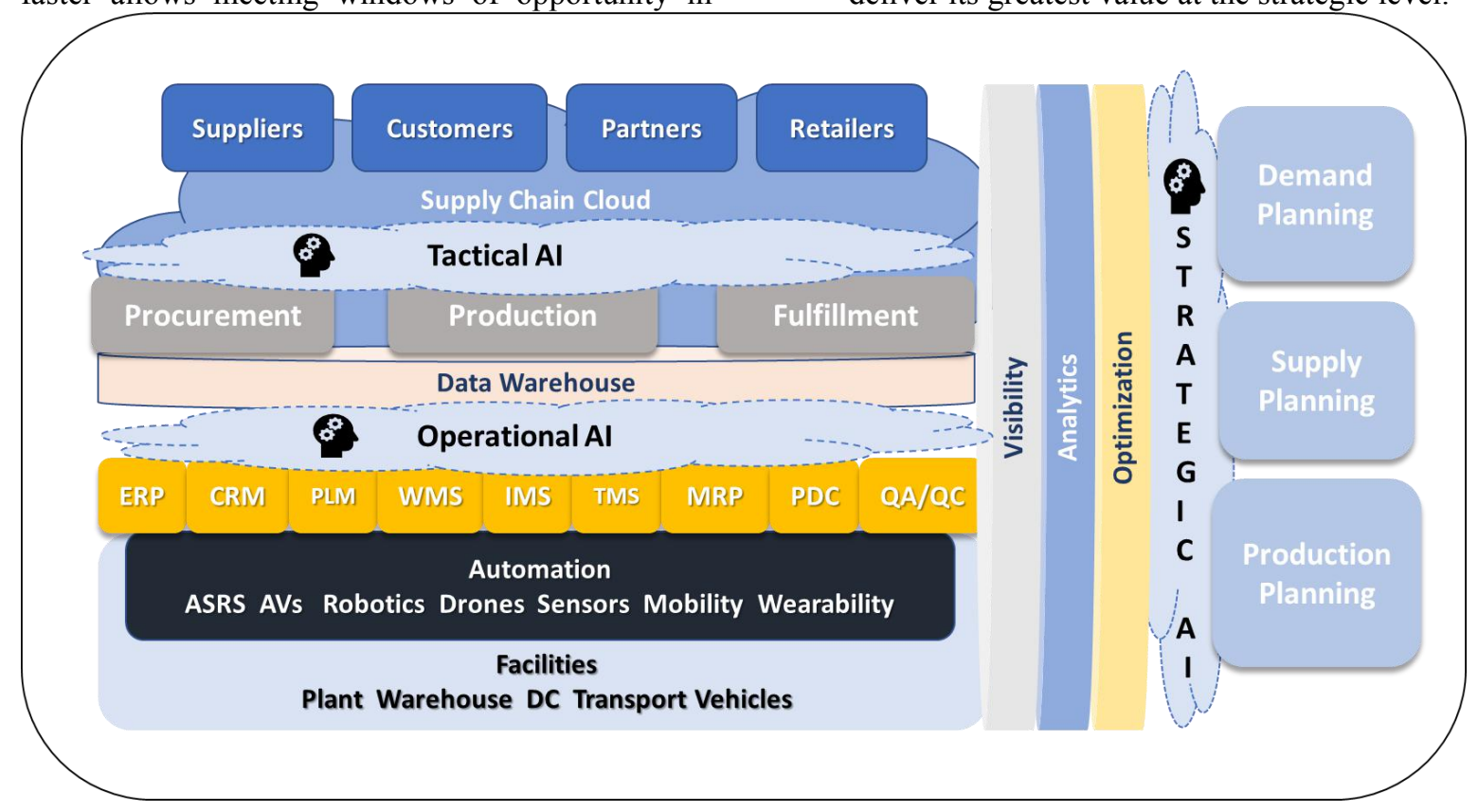

Figure 2. AI Placement within the CT Environment

\subsection{Success Factors}

Many companies are lagging behind when it comes to the application of new technologies, such as machine learning and AI, across and within business sectors. Healthcare, including pharmaceuticals, is behind in adoption of AI solutions relative to other sectors (18) . The reasons for this center on the financial risk related to the uncertainty in their efficacy and fear of regulatory authorities questioning the intent of their application. Gradually integrating these technologies within the industry via adjacent markets such as transportation, logistics and financial services could ease these risks. The following are some recommended steps for successful deployment:

- Executive Sponsorship - Technology is not the critical path for effective deployment of CT technology. Instead, it begins with executive sponsorship that spans both business and technology.

- Value-based Cases - Finding an initial use case for a technology deployment is a critical step. These should involve value-based cases for deployment, perhaps a business segment with high value or business impact where demonstrated success in a given technology can serve as a role model for subsequent implementations.

- Avoiding Pitfalls - Starting with a technology and using a "build it and they will come strategy" is rarely successful. While technology first strategies such as those seen in data lake, predictive, cognitive, or digitization implementations can receive strong executive sponsorship, they can be difficult to justify and have lengthy time-to-value, at which point they can be become outdated. Furthermore, requiring a sophisticated enterprise system with high data quality at the outset is expensive, can create risk and only delay implementation.

- Process Centric Vision - The industry trend towards increased supply chain complexity evolves around three areas of outcome: enablement, effectiveness, and earnings.

- Enablement - Enabling real-time decisions and KPI's using cloud and analytics, as an example. Enablement can be classified into various areas with the prime ones being demand visibility, inventory visibility and freight analytics.

- Effectiveness - Integrating systems that characterize process methods with analytical models could help prescribe new products and 
performance improvement measures. For example, statistical analytical methods can build on real-time measurements to monitor and correct process conditions before adverse events occur, such as in the area of quality. Or, requiring that all essential systems are connected to avoid information gaps such as having a full view of inventory on-hand prior to ordering can avoid erroneous decisions.

- Earnings - A common CT platform that can integrate enterprise and legacy systems

can enable a total delivered cost analytic approach. By applying logic to various company data, details of cost and profit associated with which customers can be unveiled.

- $\quad$ Phased Rollout - Many pharmaceutical companies will have the technology basis for implementing CT capabilities. The challenge will be to identify an initial starting opportunity and developing a decision process which might differ drastically from legacy processes. A phased or rolling implementation of one type of technology at a time across the network is preferred. This can begin with those products, customers or lanes that require deeper levels of visibility and employing a combination of technologies.

\section{Reliance on AI Technology for CT Decision Making}

Decision technologies, such as machine learning and AI, can play an important role in supporting CT operations (19). The level of reliance on these technologies for decision making was explored, with regard to how these technologies can be incorporated into CT decision functions at the operational, tactical and strategic levels, along with the level of data or information quality necessary for effective decision-making in order to use them. In addition to working with tangible data and information, the intangible information that needs to be captured by these decision technologies and how could it be captured was reviewed. The intention here was to arrive at a sense of the degree to which software/system vendors have been able to instill these capabilities within their CT solutions for effective decision making.

\subsection{Implementation within}

\section{Decision-Making}

The supply CT will rely on big data analytics to drive the acquisition, cleaning, and harmonization of data from multiple sources across the entire demand network in real time through cloud technology. It should monitor current activity and enable what-if simulations and predictive analytics. Since AI and machine learning technologies are in their early stages, they should be first vetted in parallel against traditional methods within narrow segments of applications such as products or customers. Some segments should be targeted for AI automation and others for assessing more human-driven analyses, since AI and machine learning can capture human-driven information, such as social sentiment and megatrends.

Areas of decision application include traceability, sustainability and compliance. Ripe decision areas of AI application are with respect to demand sensing and evaluating transport arrival times:

- Demand sensing - factors such as weather, smog status, health of population and competitor information could drive the level of sales in a market segment or therapy area. Thus, the information accuracy and relevancy must be of the highest order to enable accurate AI algorithms.

- Transport arrival time - factors such as weather, dock status and expected deliveries per day, which represent data not within control, must be connected with data that is within control.

Used in conjunction within a CT platform, AI should enable the following:

- decision agility to respond to external events

- decision agility with changes in the competitive landscape

- $\quad$ strong competencies to execute new courses of action

- effective collaboration internally and externally

- reactive response to exceptions or when important issues arise

In terms of the analytical engine, the CT should maintain the following capabilities:

- Descriptive engine: Simulation, machine learning classification models such as random forest, k-neighbors, classification trees, k- mean clustering.

- Predictive engine: time series analysis, regression, 
and machine learning models such as principal component analysis.

- Prescriptive engine: optimization models.

Implementation of these capabilities within a $\mathrm{CT}$ should progress on how these technologies evolve as minimal viable capabilities, with the ultimate goal of deploying these as auto-pilot functions. Several levels of evolution are envisioned:

- Basic level: if-then-act modes of operation in response to pre-defined events, somewhat representative of most ERP capabilities.

- Operational level: AI knows how to run multiple scenarios and is able to mimic the operations and supply chain execution functions.

- Strategic level: making critical decisions as to where to source, what agreements are best, or choosing lowest cost highest quality option.

The operational levels are viewed as capabilities which would need to be customized to the company. A major strategic challenge is to learn optimal behavior based on both transactional data and actions of managers, while correcting for past sub-optimal decisions. Transactional, master, and financial data are the baseline components needed for tactical AI. AI intuition will require a probabilistic model of decision-making versus deterministic. The AI will have to decide whether less precise or intangible information, such as human behavior, weather, demographics, location and IoT, combined with tangible information is good enough to make a complex decision or if it is worth the risk or opportunity cost to make it without them. This would also include knowledge regarding competitor or counterpart decision behavior in response to an event.

\section{Applications of $\mathrm{AI}$ in the $\mathrm{CT}$ Environment}

AI can thus play a valuable role in the decision-making processes within a $\mathrm{CT}$ environment. Such decision-making requires learning based on high-quality transaction-based data, less tangible data and prior human-based operator decision behavior patterns. To this end, it would be desirable to know those supply chain functions that would be best applicable for
AI or machine learning and the mechanisms that could be used to validate AI driven decisions in comparison with human-based. This involves knowing the attributes of a decision that should be used as criteria to vet $\mathrm{AI}$ decisions against those prescribed by $\mathrm{CT}$ operators, and how would such criteria could change based on the CT level (operational, tactical, strategic). The degree of reliance on AI based-decisions and how they can be used to replace or augment those coming from CT operators should be questioned. The degree of reliance could change across CT levels (20).

Furthermore, many decision-making processes require some kind of standardization, and teams will have to be forged around different technical skills and decision-making areas. This can impact the structure of the organization, and to some extent, firms would have to reorganize or reconstitute functional roles in areas of the supply chain or information technology to accommodate new kinds of decision-making. Reorganization requirements could change based on a company's role in the supply chain, such as that of producer, wholesaler, distributor or re-packager.

Supply chain planning is a function that can utilize AI and machine learning as a decision support tool. Machine-based based capabilities can boost CT provider capabilities due to the greater breadth of data from multi-client sources, versus dealing with only a single client. As a rule, the functions that are best suited for AI are basic operational functions that are currently being performed by humans. Transactional functions could be infused with added intelligence and automated, to improve accuracy, margin and ROI.

\subsection{Demand Sensing}

Demand forecasting typically involves supervised learning methods, such as regression and time series approaches. Unsupervised learning can provide opportunities where solution models are neither well defined nor intuitive. Demand can be sensed, versus forecasted, based on diverse data and information in terms of magnitude, social sentiment and trends. Demand forecasting and production planning are time intensive and quite often are not very effective. Predictive algorithms such as Principal Component and Artificial Neural Networks can be used with historical data, but 
also other data such as market trends and leading indicators.

Instead of relying on human operators to spot demand changes using dashboards or reports, multiple variables can be processed and the machine can be programmed to respond. In addition, demand patterns can be learned and adjustments made. The technology would evolve from providing visibility and alerts to expediting actions, such as replenishments.

Feedback consolidation and analytics can be used to determine consumer preferences for products such as certain kinds of pharmaceutical brands or products. This kind of demand sensing could impact inventory management and be used to improve operations. For example, AI demand patterns can be used to identify optimal product allocation to improve logistics efficiency.

Demand sensing would take into account not only previous demand data, but also information from other sources such as weather forecasts, convenience store demand, and so on. Product could be shipped sooner in anticipation of such events, thereby pushing availability to distribution partners and channels, and possibly increasing market share. In addition, as the life sciences industry grows more patient-centric, incorporating patient preferences and opinions can influence demand sensing, as consumers take control of brands through social media data content, such as blogs, forums, media posts, chats, tweets, images, videos, etc. Real-time sentiment analysis involves the recognition of text, speech via data collection, cleaning, preprocessing, and words representation, lexicon sentiment classification, and machine learning sentiment classification. The most straight forward application of social sentiment analysis would be in regard to consumer pharmaceutical products such as over-the-counter (OTC), but other potential applications would include favoring certain healthcare provider prescriptions brands and opinions on preferred drug therapies, such as vaccines.

\subsection{Inventory Management}

Risk management of inventory is another fertile area of application of AI. AI and machine learning based automated exception tools can offer several opportunities:

- General prediction of demand to improve work center and allocations;

- Sending alerts in anticipation of unexpected events, such as the next flu outbreak;

- Inventory classification algorithms for more exact $\mathrm{ABC}$ classification, with emphasis on identifying A-Category stocking units (SKUs);

- Stockout or service level prediction;

- Pragmatic approaches to procurement due to margin constraints;

- Automating basic replenishment using algorithms and automating procurement contracts,

- Identifying cross-sell/up-sell opportunities.

\subsection{Master Scheduling}

Traditional materials requirements planning (MRP) functions can be modified with AI to include factors other than order requirements, such as inventory shelf life, customer shelf life requirements, changes in lead times of suppliers, change in risk levels, human resource changes, or transportation lead time changes. Production orders can be adjusted to reflect the changes in conditions detected in these areas. Similarly, logistics planning can also be improved by using machines to evaluate the best available routes, particularly for route optimizations with regard to freight.

\subsection{Validating AI Decision Making}

Pilots should be run to compare existing decision processes with new AI based processes, in order to validate them and define their scope of deployment. The pilots could follow test-train phasing depending on the level of decision. A three-step process is envisioned:

- Predict and validate over a hold-out period: The machine must first learn how to perform a task, with rules that prevent negative outcomes.

- Predict and run parallel test with human decisions: This would involve running in parallel both machine and human based tactical decisions as decision support while the algorithm is trained. A validation protocol would need to be established that would carefully test the AI machine through many scenarios, and stress testing through normal and abnormal situations, such as power failures, upgrades to connected software or extreme inputs. Validation should be based on a set of criteria (e.g. dollar amount per day/transaction, delay for 
delivery, etc.), metrics, including significance level, area under the curve, accuracy, precision, business performance metrics and customer impact.

- Predict and run actions with human supervision/exceptions: The business should decide what decisions should be machine-based, human-based and combined, with continuous performance review to make improvements.

\subsection{Organizational Reform}

The supply chain professionals of the future will evolve from the management of exceptions to creating more strategic value through new ways of working. Firms might have to reorganize or reconstitute functional roles within supply chain and perhaps information technology to accommodate machine-based decision-making. Since many stakeholders within an organization might not equivalently value enhanced CT solutions, internal customer driven KPI's with specific business performance outcomes need to be designed and agreed upon prior to implementation.

Operators will need to trust the AI outcomes and buy-into machine-assisted decision-making. This becomes challenging in situations where consequences can be costly, such as optimal packaging runs or quota requirements on controlled substance products like opioids. This can be accomplished through the validation process by demonstrating the effectiveness of machine-based results. Reliance on AI is grounded on continuous testing of models and demonstrating the ability to trust the outcome. As algorithms get better at adapting to market changes, companies will need to become more agile, requiring greater internal collaboration.

There will also be a need for more partnerships to manage upstream/downstream commerce, ultimately necessitating standards that can link disparate data and transactional systems. Opportunities could open for third-party firms to provide a service that would consolidate the different data streams if it can integrate at least the large players in current transactional systems.

\section{Major Challenges}

For pharmaceutical companies, particularly those that work extensively with Contract Manufacturing
Organizations (CMOs), there could be major pain points, especially around data quality, maintenance and security (21) . There is much concern regarding standardized processes and integration platforms to efficiently on-board or off-board CMOs within the CT. This might require segmenting CMOs into strategic tiers and being able to measure their performance. Another question deals with the impact of the level of inter-company collaboration on CT effectiveness. Specifically, whether CMOs could benefit from data sharing within a CT environment and what mechanisms can best be used to share data between CMOs within the CT. For data sharing, the lowest levels of data that should be disclosed from the CMO within the CT would include work-in-process, inventory, materials, development data from lab information management systems (LIMS), among others.

\subsection{Data Sharing}

If CMO data is incomplete, unavailable or wrong, CTs cannot make decisions and take action on the fly. Nor could attempts at automation, machine learning and other technologies be effective. This implies foundational measures to maintain, secure and assure data quality at this level. This can be challenging when the CMO is working with different systems and version numbers and could necessitate maintaining translation tables between systems, such as ERP systems or other systems such as LIMS systems. This issue is compounded when one works with multiple CMOs, making quality conformance challenging. Using a distributed ledger involving blockchain technology, the CMO or authorizing entity could encode the batch record or the data record from inception, thus any changes in reference code could be recorded in the ledger as blockchained, which may help ease using translation tables.

Furthermore, the regulatory requirements for the product will also drive the serialization data labeling and quality requirements. If the $\mathrm{CMO}$ is dealing with a product requiring critical testing and control, the detailed data about the batch record is needed. Having this information available can improve the speed of record review and approval within the supply chain at each of the levels identified previously: 
- Operational level - AI can help isolate deviations and expedite complaint resolution, thus increasing process efficiency. When deviations are found or customer complaints are received, it also increases the efficiency of the process at the operational level.

- Tactical and strategic levels - AI can aid in the tracking of KPIs, performance indicators, electronic contracts, financial data, leading indicator data, and demand sensing data with visibility to the needed parties. This capability can improve planning beyond the planning time horizon.

While many CMOs promise sound quality, some precautions that pharmaceutical firms can take to control product quality can be through stationing quality personnel the CMO facility to implement their own quality protocols at the $\mathrm{CMO}$ and establishing their own KPIs to measure quality.

Strong collaboration with CMOs should be encouraged to acquire qualitative data as well as quantitative data. Both parties need to map out the data each requires and then work to imbed the data within the CT. At the operational level, the CMO can offer the buyer visibility to their schedules, beyond the frozen time fence and to any planned campaigns to support the buyer needs. The buyer might have to provide data such as inventory levels, order loading, historical trends, among others, which is information the CMO may require for the purposes of capacity planning. At the tactical level, quality metrics, audit actions and other information can be shared. At the strategic level, data regarding longer time horizons can also be shared (22).

\subsection{Data Visibility}

CMOs have a significant impact on CT upstream visibility, since horizontal integration can decrease supply chain visibility. It may also require more intercompany processes, which can be difficult to manage and integrate. Segmentation by volume or profit can be useful to manage the added complexity, but should be done carefully since some CMOs are typically owned by or outsourced to wholesalers. It can help determine which CMOs are important to integrate and identify the necessary flows between, people, systems and processes. Some approaches that can be used to ensure visibility and compliance include:

- Establishing contracts having predefined operational requirements for processes and systems;

- Developing a clear work plan with proper project management to ensure synchronized operations;

- For important CMOs, creating the system interoperability for transactional (e.g. stock transfers, purchase orders, sales orders) and planning systems.

Among the many challenges related to data sharing with CMOs would lie in communicating their demand plans for highly sensitive products, such as opioids, that have to be conveyed to regulatory agencies. Tracking actual versus forecasted demand with pre-established quotas would be necessary. CMOs would become burdened with having to integrate their systems to provide real time information, and meeting serialization and tracking requirements. Adequate KPIs would need to be established with CMOs to convey site-to-site and batch-to-batch data comparisons, data access, sharing, and retrieval, and quality assurance. Furthermore, the methods used for KPI measurement and reporting output need to be agreed upon by the involved parties.

\section{Conclusions}

Today's CT solutions provide somewhat simplistic, limited visibility while claiming end to end visibility, providing a window into one part of the supply chain at the expense of the other. Visibility boils down to the execution of orders while balancing costs and availability to satisfy contracts and customer demand. In this respect, the CT must provide a holistic end-to-end view across supply chain suppliers, contract manufacturers, transportation carriers, and third-party logistic vendors to track the active movements of goods from origin to destination. This should entail visibility beyond just 1 or 2 tiers, depending on the industry, and enabling technology at different levels of automation. It should enable peering into channels to view excess and expiring inventory from a central node, ultimately to aid in smoothing operations and reducing bullwhip effects.

Technologies such as AI can play a valuable role in the decision-making processes within a CT environment. Such decision-making requires learning based on high-quality transaction-based data, less tangible data and prior human-based operator decision behavior 
patterns. The supply chain professionals of the future will evolve from the management of exceptions to creating more strategic value through new ways of working. Firms might have to reorganize or reconstitute functional roles within supply chain and perhaps information technology to accommodate machine-based decision-making.

\section{References}

1. Trzuskawska-Grzesińska A. Control Towers in Supply Chain Management - Past and Future. Journal of Economics and Management. 2017; 27 (1): p. 117-119.

2. Linton JD. Emerging Technology Supply Chains. Technovation. 2017;: p. 1-3.

3. MPO Objects. Modern Control Towers: Choosing the Right One for Your Digital Supply Chain. White Paper.; 2017.

4. Baumgrass A, Dijkman R, Grefen P, Pourmirza S, Völzer H, Weske M. A Software Architecture for a Transportation Control Tower. Beta Working Paper series 461. Beta Research School for Operations Management and Logistics; 2014.

5. Hofman W. Control Tower Architecture for Multi and Synchromodal. In ILS 2014 5th International Conference; 2014; Breda.

6. Mabert VA, Venkataraamanan MA. Special Research Focus on Supply Chain Linkages: Challenges for Design and Management in the 21st Centruy. Decision Sciences. ; 29(3): p. 537-552.

7. Accenture. Prepare for Takeoff with a Supply Chain Control Tower. ; 2014.

8. Trzuskawska-Grzesińska A. Control Towers in Supply Chain Management - Past and Future. Journal of Economics and Management. 2017; 27(1): p. 117-124.

9. Lee BK, Zhou R, deSouza R, Park J. Data-Driven Risk Measurement o fFirm-to-Firm Relationships in a Supply Chain. International Journal of Production Economics. 2016; 180: p. 148-157.

10. Singh RK, Kumar R, Kumar P. Strategic Issues in Pharmaceutical Supply Chains: A Review. International Journal of Pharmaceutical and Healthcare Marketing. 2016; 10(3): p. 234-257.

11. Accenture. Creating a Supply Chain Control Tower in the High-Tech Industry. ; 2015.

12. Hansen KR, Grunow M. Planning Operations Before Market Launch for Balancing Time-to-Market and Risks in Pharmaceutical Supply Chains. International Journal of Production Economics. 2015; 161: p. 129-139.

13. Chen X, Yang H, Wang X. Effects of Price Cap Regulation on the Pharmaceutical Supply Chain. Journal of Business Research. 2018;: p. 1-3.

14. Papert M, Rimpler P, Pflaum A. Enhancing Supply
Chain Visibility in a Pharmaceutical Supply Chain. International Journal of Physical Distribution \& Logistics Management. 2016; 46(9): p. 859-884.

15. Liotine M, Pagano A, Gadiraju SV. Technology Trends in Logistics and Supply Chain Management. In Pagano AM, Gyimah M. Contemporary Issues in Supply Chain Management and Logistics. New York: Business Expert Press; 2017. p. 6-29.

16. Swaminathan J, Smith SF, Sadeh NM. Modeling Supply Chain Dynamics: A Multi-Agent Approach. Decision Sciences. 1998; 29(3): p. 607-631.

17. Wu DJ, Zhong F, Kimbrough SO. Computers Play the Beer Game: Can Artificial Agents Manage Supply Chains? Decision Support Systems. 2002; 33: p. 331-332.

18. Bughin J, Hazan E, Ramaswamy S, et. al. Artifical Intelligence: The Next Digital Frontier. Discussion Paper. McKinsey Global Institute; 2017.

19. Lippincott S. Control Tower Technology Value Matrix 2017. Research Note. Nucleus Research; 2017.

20. Fox M, Barbuceanu M, Teigen R. Agent-Oriented Supply-Chain Management. The International Journal of Flexible Manufacturing Systems. 2000; 12: p. 165-188.

21. Shah N. Pharmaceutical Supply Chains: Key Issues and Strategies for Optimisation. Computers and Chemical Engineering. 2004; 28: p. 929-941.

22. A Composite Framework of Supply Chain Management and Enterprise Planning for Small and Medium Sized Manufacturing Enterprises. Supply Chain Management: An International Journal. 2008; 13(5): p. 349-355. 